\title{
New asymptotic description of nonlinear water waves in Lagrangian coordinates
}

\author{
By E. V. BULDAKOV ${ }^{1}$, P. H. TAYLOR \\ AND R. EATOCK TAYLOR \\ ${ }^{1}$ Department of Civil Engineering, University College London, WC1E 6BT, UK \\ ${ }^{2}$ Department of Engineering Science, University of Oxford, OX1 3PJ, UK
}

(Received 2 May 2004 and in revised form 6 March 2006)

A new description of two-dimensional continuous free-surface flows in Lagrangian coordinates is proposed. It is shown that the position of a fluid particle in such flows can be represented as a fixed point of a transformation in $\mathbb{R}^{2}$. Components of the transformation function satisfy the linear Euler-type continuity equation and can be expressed via a single function analogous to an Eulerian stream function. Fixedpoint iterations lead to a simple recursive representation of a solution satisfying the Lagrangian continuity equation. Expanding the unknown function in a smallperturbation asymptotic expansion we obtain the complete asymptotic formulation of the problem in a fixed domain of Lagrangian labels. The method is then applied to the classical problem of a regular wave travelling in deep water, and the fifthorder Lagrangian asymptotic solution is constructed, which provides a much better approximation of steep waves than the corresponding Eulerian Stokes expansion. In contrast with early attempts at Lagrangian regular-wave expansions, the asymptotic solution presented is uniformly valid at large times.

\section{Introduction}

The main complication of problems with a free surface is that the Eulerian position of this surface, being the boundary of the solution domain, is not specified a priori, and must be found as a part of the solution. The application of the Lagrangian description of fluid motion is the natural way to overcome this difficulty. For a wide class of flows the free surface remains a simply connected domain and is free of singularities. In such cases surface particles stay on the surface throughout the entire motion, which means that in the Lagrangian description the free surface is represented by a fixed boundary of the domain in the space of Lagrangian labels.

Most previous studies of the Lagrangian properties of water waves and their practical applications do not refer directly to the Lagrangian equations of fluid motion, but rather solve the Eulerian equations and afterward calculate particle motion (e.g. Longuet-Higgins 1979, 1986, 1987; McIntyre 1988). There are only a few published cases where Lagrangian equations are directly applied to solve water wave problems. A small-perturbation analysis of the equations of fluid motion in Lagrangian form was performed by Pierson (1962), who represented the displacement of fluid particles as a regular expansion with respect to powers of a small parameter. Selected first-order solutions, including those with a free surface, were constructed and a general second-order formulation was presented. The well-known Gerstner solution (e.g. Lamb 1932) for a regular travelling wave in deep water can be formally obtained as a first-order Lagrangian asymptotic solution for small wave steepness. 
Although Gerstner's waves are not irrotational, for wave steepness approaching zero the vorticity contribution is asymptotically small compared to contribution of other effects. Gjøsund (2003) considered first-order Lagrangian solutions for irregular waves in water of finite depth. The second-order Lagrangian asymptotic analysis was successfully applied by Ünlüata \& Mei (1970) to the problem of mass transport by a regular wave. However, Pierson-type expansions have not found wide application. A contributing reason may be the nonlinearity of the Lagrangian continuity equation. In the case of incompressible flow the Eulerian continuity equation is linear, and it is exactly satisfied by an asymptotic expansion of any order. On the other hand, such an expansion satisfies the nonlinear Lagrangian continuity equation only approximately, with unbalanced higher-order source terms appearing in the solution. This leads to the violation of the most fundamental property of mass conservation. As a result, the third- and higher-order Pierson-type expansions for a regular travelling wave are nonuniformly valid for large times. We resolve this difficulty for two-dimensional flows, and propose an asymptotic description which exactly satisfies the two-dimensional Lagrangian continuity equation.

In $\S 2$ we give a general formulation for two-dimensional waves in Lagrangian coordinates. In $\S 3$ we show that the Lagrangian position of a fluid particle in a continuous two-dimensional flow is a fixed point of a certain transformation in $\mathbb{R}^{2}$. The components of the transformation function satisfy the Eulerian linear continuity equation and can be expressed via a single function similar to a stream function in the Eulerian description. Fixed-point iterations lead to a simple recursive representation of the solution. In $\S 4$ we represent the unknown function as an asymptotic expansion for small wave steepness (small volume deformation) and obtain the complete asymptotic formulation of the problem in a fixed domain of Lagrangian labels. As a test case we consider the problem of a regular travelling wave in deep water. An application of the small-perturbation technique to this problem in Eulerian form leads to the classical Stokes expansion (see Stokes 1847 and Schwartz \& Fenton 1982 for a review of the Stokes expansion and related results). In $\S 5$ we first consider the Pierson-type approach to the problem and demonstrate that the third- and higher-order solutions are non-uniformly valid at large time. Then we construct a uniformly valid solution using the novel recursive representation. The expansion is taken to fifth order only, yet succeeds in calculating the wave shape close to maximum steepness. Section 6 offers some concluding remarks.

\section{Lagrangian formulation for two-dimensional free-surface flows}

Let $x=x(a, c, t), z=z(a, c, t)$ be Cartesian coordinates of a fluid particle marked by Lagrangian labels $(a, c)$ at the time $t$. For incompressible fluid the Jacobian $J$ of the mapping $(a, c) \rightarrow(x, z)$ is the motion invariant and Lagrangian labels can be chosen in such a way that

$$
J=\frac{\partial(x, z)}{\partial(a, c)}=1 .
$$

This is the case when labels $(a, c)$ are physically possible coordinates $(x, z)$ of a particle, e.g. the coordinates at a certain reference time $t_{0}$ or equilibrium coordinates: $(a, c)=\left(x_{0}, z_{0}\right)$. The equations of the dynamics of an inviscid fluid can then be written as (Lamb 1932)

$$
\frac{\partial P}{\partial a}+g z_{a}=-x_{t t} x_{a}-z_{t t} z_{a}, \quad \frac{\partial P}{\partial c}+g z_{c}=-x_{t t} x_{c}-z_{t t} z_{c},
$$


where $P$ is the ratio of pressure over density. The terms on the left-hand sides of (2.2) are gradient components of a scalar function in the label space. Taking the curl of both sides of (2.2) we find that

$$
\Omega=\nabla_{a} \times\left(x_{t} x_{a}+z_{t} z_{a}, x_{t} x_{c}+z_{t} z_{c}\right)
$$

is the motion invariant: $\partial \Omega / \partial t=0$, where $\nabla_{a} \times$ is the curl operator in $(a, c)$-space. This is the Lagrangian form of vorticity conservation (e.g Salmon 1988). Thus, we have another kinematic condition in addition to (2.1):

$$
\frac{\partial\left(x_{t}, x\right)}{\partial(a, c)}+\frac{\partial\left(z_{t}, z\right)}{\partial(a, c)}=\Omega(a, c)
$$

where the vorticity $\Omega(a, c)$ is a given function of the Lagrangian labels. A prescribed pressure distribution at $c=0$ provides a condition on the water surface, and in the case of a free surface ( $P=$ const) equation $(2.2 a)$ gives

$$
x_{t t} x_{a}+z_{t t} z_{a}+\left.g z_{a}\right|_{c=0}=0 .
$$

The complete formulation consists of the continuity equation (2.1), the vorticity conservation equation (2.3) and the free-surface condition (2.4), plus proper conditions on rigid and open boundaries.

Hereafter we apply non-dimensional variables using the same notation as before. We use a characteristic wavenumber $k$ to scale physical coordinates and Lagrangian labels, a characteristic wave amplitude $\varepsilon$ for scaling particle displacement from the equilibrium, and a characteristic frequency $\omega$ to scale time.

\section{Kinematics: recursive representation}

In this section we consider mappings $(a, c) \rightarrow(x, z)$ satisfying the continuity condition (2.1). The time dependence is irrelevant at this stage and we omit the time variable, assuming, however, that all functions depend on time as a parameter.

Let us write the Cartesian position of a fluid particle in the following form:

$$
x(a, c)=a+k \varepsilon \xi(\alpha, \gamma), \quad z(a, c)=c+k \varepsilon \zeta(\alpha, \gamma),
$$

where $k \varepsilon$ is the non-dimensional amplitude of the displacement, and the displacement vector $(\xi, \zeta)$ of a particle from the reference position $\left(x_{0}, z_{0}\right)=(a, c)$ is a function of new variables

$$
\alpha=\frac{1}{2}(a+x(a, c)), \quad \gamma=\frac{1}{2}(c+z(a, c)) .
$$

Thus $(\alpha, \gamma)$ is the mean of the current and the equilibrium position of a particle. The particle position and the corresponding Lagrangian labels as functions of $(\alpha, \gamma)$ are

$x=\alpha+k \varepsilon \frac{1}{2} \xi(\alpha, \gamma), \quad z=\gamma+k \varepsilon \frac{1}{2} \zeta(\alpha, \gamma) ; \quad a=\alpha-k \varepsilon \frac{1}{2} \xi(\alpha, \gamma), \quad c=\gamma-k \varepsilon \frac{1}{2} \zeta(\alpha, \gamma)$.

We can represent $J$ as the ratio of two Jacobians

$$
J=\frac{\partial(x, z)}{\partial(\alpha, \gamma)} / \frac{\partial(a, c)}{\partial(\alpha, \gamma)},
$$

where

$$
\begin{aligned}
& \frac{\partial(x, z)}{\partial(\alpha, \gamma)}=1+k \varepsilon \frac{1}{2}\left(\xi_{\alpha}+\zeta_{\gamma}\right)+(k \varepsilon)^{2} \frac{1}{4}\left(\xi_{\alpha} \zeta_{\gamma}-\xi_{\gamma} \zeta_{\alpha}\right), \\
& \frac{\partial(a, c)}{\partial(\alpha, \gamma)}=1-k \varepsilon \frac{1}{2}\left(\xi_{\alpha}+\zeta_{\gamma}\right)+(k \varepsilon)^{2} \frac{1}{4}\left(\xi_{\alpha} \zeta_{\gamma}-\xi_{\gamma} \zeta_{\alpha}\right) .
\end{aligned}
$$


Thus since $J=1$, functions $\xi$ and $\zeta$ satisfy a linear form of the continuity equation:

$$
\frac{\partial \xi}{\partial \alpha}+\frac{\partial \zeta}{\partial \gamma}=0
$$

Hence they can be expressed via a single function $\Psi(\alpha, \gamma)$ analogous to a stream function of the Eulerian description:

$$
\xi(\alpha, \gamma)=-\frac{\partial \Psi}{\partial \gamma}, \quad \zeta(\alpha, \gamma)=\frac{\partial \Psi}{\partial \alpha} .
$$

The Jacobians in the numerator and denominator of (3.3) become

$$
\frac{\partial(x, z)}{\partial(\alpha, \gamma)}=\frac{\partial(a, c)}{\partial(\alpha, \gamma)}=(k \varepsilon)^{2} \frac{1}{4}\left(\Psi_{\alpha \alpha} \Psi_{\gamma \gamma}-\Psi_{\alpha \gamma}^{2}\right)+1 .
$$

We may think of the mapping $(a, c) \rightarrow(x, z)$ as the combination of two mappings, $(a, c) \rightarrow(\alpha, \gamma)$ followed by $(\alpha, \gamma) \rightarrow(x, z)$. Such a combination may become singular if the numerator and denominator in (3.3) become zero, i.e.

$$
(k \varepsilon)^{2} \frac{1}{4}\left(\Psi_{\alpha \alpha} \Psi_{\gamma \gamma}-\Psi_{\alpha \gamma}^{2}\right)+1=0,
$$

as is illustrated by an example below in this section.

Equations (3.1) and (3.2) imply that the transformed variables $(\alpha, \gamma)$ are defined by a fixed point of a mapping

$$
\alpha=a+k \varepsilon \frac{1}{2} \xi(\alpha, \gamma), \quad \gamma=c+k \varepsilon \frac{1}{2} \zeta(\alpha, \gamma),
$$

and fixed-point iterations starting from a suitable initial approximation can be used to find them. It is convenient to take $(\alpha, \gamma)=(a, c)$ as an initial approximation. Then the repeated application of (3.7) leads to the following recursive sequence:

$$
\left.\begin{array}{l}
x_{m}(a, c)=\alpha_{0}+k \varepsilon \xi\left(\alpha_{m}, \gamma_{m}\right), \quad z_{m}(a, c)=\gamma_{0}+k \varepsilon \zeta\left(\alpha_{m}, \gamma_{m}\right) ; \\
\alpha_{0}=a, \quad \gamma_{0}=c ; \\
\alpha_{m}=\alpha_{0}+k \varepsilon \xi\left(\alpha_{m-1}, \gamma_{m-1}\right) / 2, \quad \gamma_{m}=\gamma_{0}+k \varepsilon \zeta\left(\alpha_{m-1}, \gamma_{m-1}\right) / 2 .
\end{array}\right\}
$$

If the sequence $\left(x_{m}, z_{m}\right)$ converges to $(x, z)$ as $m \rightarrow \infty$ for a certain domain in $(a, c)$, the continuity condition (2.1) for a mapping $(a, c) \rightarrow(x, z)$ is satisfied in this domain.

We consider a simple example. Let the function $\Psi$ be written

$$
\Psi(\alpha, \gamma)=\mathrm{e}^{\gamma} \sin (\alpha) .
$$

This function can be used as a leading approximation of small-amplitude asymptotic solutions for standing (variable amplitude) or travelling (variable phase) waves in deep water. For a one-dimensional mapping $f(x)$ the condition of convergence of fixed-point iterations $x_{m}=f\left(x_{m-1}\right)$ is $f^{\prime}(x)<1$. This condition can be applied on a straight rigid boundary or on a symmetry axis, when a point from a line will always be mapped into another point of the same line. For our model solution (3.9) such a line is $x=a=\alpha=0$, and we expect that the convergence condition first breaks down on the free surface $c=0$. Applying the one-dimensional convergence condition to the second component of (3.7) for $c=0$ we obtain

$$
f(\gamma)=k \varepsilon \frac{1}{2} \mathrm{e}^{\gamma}=\gamma, \quad f^{\prime}(\gamma)=k \varepsilon \frac{1}{2} \mathrm{e}^{\gamma}=1 .
$$

Solving these equations we find that the convergence condition breaks down when

$$
k \varepsilon=\frac{2}{e}=0.73575 \ldots, \quad \gamma=1,
$$



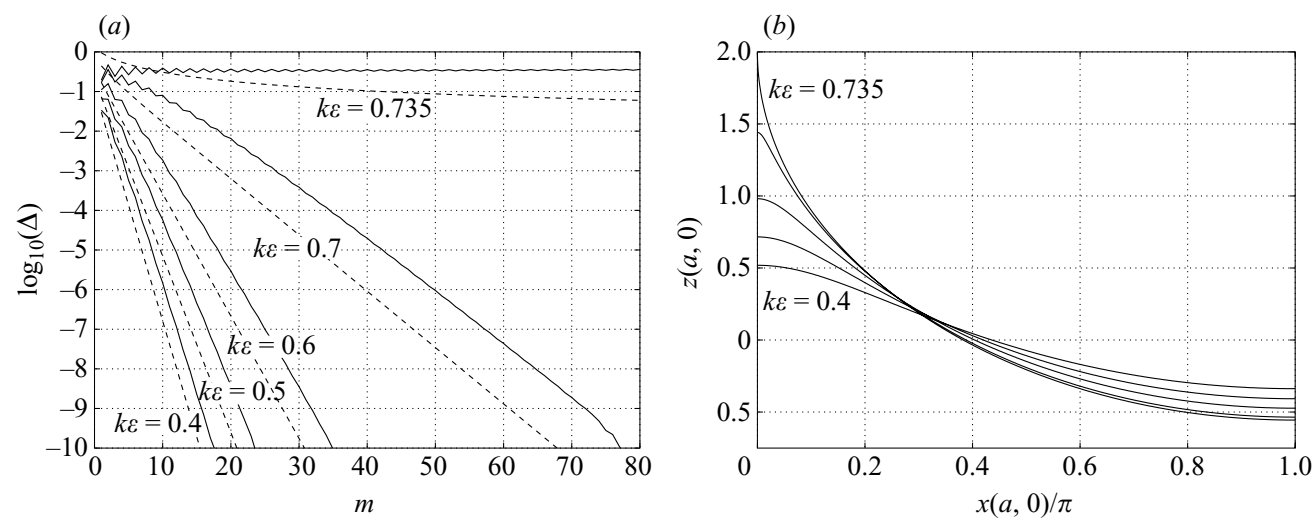

Figure 1. (a) The convergence of the recursion (3.8) for $z(0,0)$ for the model solution (3.9) with various values of $k \varepsilon . \Delta=J-1$ for solid lines, and $\Delta=\left|z_{m}-z_{500}\right|$ for dashed lines. (b) The corresponding profiles for $c=0$.

and the corresponding surface elevation is $z(0,0)=2$. The condition (3.6) for regularity of the mapping $(a, c) \rightarrow(\alpha, \gamma)$ also breaks down at this point. The curve $c=0$ representing the free surface of our model solution has the following parametric representation in physical coordinates:

$$
x=-\frac{k \varepsilon \mathrm{e}^{\gamma}}{2} \sqrt{1-\frac{4 \gamma^{2}}{(k \varepsilon)^{2} \mathrm{e}^{2 \gamma}}}+\arccos \left(\frac{2 \gamma}{k \varepsilon \mathrm{e}^{\gamma}}\right), \quad z=2 \gamma .
$$

For the critical value of $k \varepsilon$ and $\gamma=1$ we have $x=0, z=2$. The slope becomes infinite at this point:

$$
\frac{\mathrm{d} z}{\mathrm{~d} x}=-\frac{1}{\gamma-1}+\frac{2(\gamma-1)}{9}-O(\gamma-1)^{2} \quad \text { as } \quad \gamma \rightarrow 1, \quad \gamma<1 .
$$

The results of computation of the recursive solutions (3.8) for the model function (3.9) with various amplitudes $k \varepsilon$ are presented in figure 1 . The convergence of iterations is fast for small and moderate amplitudes. For $k \varepsilon=0.7$, which is only $5 \%$ less than the critical amplitude, the solution satisfies the Lagrangian continuity equation (2.1) with accuracy $10^{-10}$ after about 80 iterations.

We have therefore shown that a solution of the form (3.2), (3.1), (3.5) satisfies the Lagrangian continuity equation (2.1) and can be constructed by applying the recursive procedure (3.8). Function $\Psi(\alpha, \gamma)$ is an arbitrary differentiable function and its particular form must be found from the equation of vorticity conservation (2.3) and the boundary conditions. In the following section we perform this analysis by applying a small-perturbation technique for the case of small local deformations of fluid volume.

\section{Dynamics: asymptotic representation}

We assume the scale of particle displacement $\varepsilon$ to be small compared to the characteristic wavelength and consider the limit $k \varepsilon \rightarrow 0$. After expansion with respect to the small parameter $k \varepsilon$ all functions appear as functions of Lagrangian labels $(a, c)$. Let us consider the Jacobian $J_{m}$ of the transformation $(a, c) \rightarrow\left(x_{m}, z_{m}\right)$ corresponding to the $m$ th level of the recursion (3.8). Similarly to the previous section we consider 
this transformation as a combination of two transformations $(a, c) \rightarrow\left(\alpha_{m}, \gamma_{m}\right)$ and $\left(\alpha_{m}, \gamma_{m}\right) \rightarrow\left(x_{m}, z_{m}\right)$, and represent $J_{m}$ as a product of their Jacobians:

$$
J_{m}=\frac{\partial\left(x_{m}, z_{m}\right)}{\partial(a, c)}=\frac{\partial\left(x_{m}, z_{m}\right)}{\partial\left(\alpha_{m}, \gamma_{m}\right)} / \frac{\partial(a, c)}{\partial\left(\alpha_{m}, \gamma_{m}\right)} \text {. }
$$

By induction one can easily prove that for regular functions $\xi$ and $\zeta$ the following asymptotic relations are valid:

$$
\begin{aligned}
& \alpha_{m}=\alpha_{m-1}+(k \varepsilon)^{m} A_{m}\left(\alpha_{m}, \gamma_{m}\right)+O(k \varepsilon)^{m+1}, \\
& \gamma_{m}=\gamma_{m-1}+(k \varepsilon)^{m} C_{m}\left(\alpha_{m}, \gamma_{m}\right)+O(k \varepsilon)^{m+1},
\end{aligned}
$$

where functions $A_{m}$ and $C_{m}$ are $O(1)$ as $k \varepsilon \rightarrow 0$. Applying this to (3.8) we have

$$
\begin{aligned}
x_{m} & =\alpha_{m}+k \varepsilon \xi\left(\alpha_{m}, \gamma_{m}\right) / 2+(k \varepsilon)^{m+1}\left(A_{m} \xi_{\alpha}+C_{m} \xi_{\gamma}\right)+O(k \varepsilon)^{m+2}, \\
z_{m} & =\gamma_{m}+k \varepsilon \zeta\left(\alpha_{m}, \gamma_{m}\right) / 2+(k \varepsilon)^{m+1}\left(A_{m} \zeta_{\alpha}+C_{m} \zeta_{\gamma}\right)+O(k \varepsilon)^{m+2}, \\
a & =\alpha_{m}-k \varepsilon \xi\left(\alpha_{m}, \gamma_{m}\right) / 2+(k \varepsilon)^{m+1}\left(A_{m} \xi_{\alpha}+C_{m} \xi_{\gamma}\right)+O(k \varepsilon)^{m+2}, \\
c & =\gamma_{m}-k \varepsilon \zeta\left(\alpha_{m}, \gamma_{m}\right) / 2+(k \varepsilon)^{m+1}\left(A_{m} \zeta_{\alpha}+C_{m} \zeta_{\gamma}\right)+O(k \varepsilon)^{m+2},
\end{aligned}
$$

and the corresponding asymptotic expansions for Jacobians in both the numerator and denominator of (4.1) are

$$
1+(k \varepsilon)^{2} \frac{1}{4}\left(\Psi_{\alpha \alpha} \Psi_{\gamma \gamma}-\Psi_{\alpha \gamma}^{2}\right)+(k \varepsilon)^{m+1}\left(\left(A_{m} \xi_{\alpha}+C_{m} \xi_{\gamma}\right)_{\alpha}+\left(A_{m} \zeta_{\alpha}+C_{m} \zeta_{\gamma}\right)_{\gamma}\right)+O(k \varepsilon)^{m+2} \text {. }
$$

The ratio of these expansions gives

$$
J_{m}=1+O(k \varepsilon)^{m+2} .
$$

Therefore, functions $x_{m}$ and $z_{m}$ satisfy the Lagrangian continuity equation (2.1) with an accuracy $O(k \varepsilon)^{m+2}$ as $k \varepsilon \rightarrow 0$. The recursive form (3.8) can be treated in two ways: (i) as an approximate asymptotic solution for $k \varepsilon \rightarrow 0$ if we take a finite number of recursion steps or (ii) as an exact solution if the recursion converges for $m \rightarrow \infty$.

To satisfy the vorticity conservation (2.3) and the free-surface condition (2.4), we represent $\Psi$ as an expansion for small $k \varepsilon$ :

$$
\Psi=\Psi_{0}+k \varepsilon \Psi_{1}+(k \varepsilon)^{2} \Psi_{2}+\cdots .
$$

For irrotational flow $\Omega=0$, the leading approximation of (2.3) is found to be

$$
\frac{\partial}{\partial t} \nabla^{2} \Psi_{0}=0
$$

which means that $\Psi_{0}$ is the sum of a harmonic function depending on time as a parameter and an arbitrary function of coordinates. It can be shown that for our choice of the Lagrangian labels the arbitrary function of coordinates is also harmonic. Let $\Psi_{0}=\Psi_{01}(a, c, t)+\Psi_{02}(a, c)$, where $\nabla^{2} \Psi_{01}=0$. Then in the leading approximation

$$
x=a-k \varepsilon \frac{\partial}{\partial c}\left(\Psi_{01}(a, c, t)+\Psi_{02}(a, c)\right), \quad z=c+k \varepsilon \frac{\partial}{\partial a}\left(\Psi_{01}(a, c, t)+\Psi_{02}(a, c)\right) .
$$

At $t=t_{0}$ we have $x=a, z=c$ and

$$
\Psi_{01}\left(a, c, t_{0}\right)+\Psi_{02}(a, c)=F(a) \quad \text { and } \quad \Psi_{01}\left(a, c, t_{0}\right)+\Psi_{02}(a, c)=G(c) .
$$

With arbitrary $F$ and $G$ this is possible only if $F(a)=G(c)=$ const. Thus, $\Psi_{02}$ is a harmonic function of $(a, c)$, and $\Psi_{0}$ satisfies the Laplace equation

$$
\nabla^{2} \Psi_{0}=0 .
$$


For higher-order terms we have equations of the form

$$
\frac{\partial}{\partial t} \nabla^{2} \Psi_{n}=R_{n}(a, c, t)
$$

with the right-hand sides $R_{n}$ depending on previous approximations. For example,

$$
R_{1}=2\left(\frac{\partial}{\partial t}\left(\frac{\partial^{2} \Psi_{0}}{\partial a \partial c}\right) \frac{\partial^{2} \Psi_{0}}{\partial a^{2}}-\frac{\partial^{2} \Psi_{0}}{\partial a \partial c} \frac{\partial}{\partial t}\left(\frac{\partial^{2} \Psi_{0}}{\partial a^{2}}\right)\right)
$$

After an integration with respect to time we obtain a Poisson equation for each $\Psi_{n}$. On a free surface $c=0$ each of the $\Psi_{n}$ satisfies a condition obtained after expansion of (2.4). For $n=0$ we have

$$
\mathscr{W}^{2} \frac{\partial^{2}}{\partial t^{2}} \frac{\partial \Psi_{0}}{\partial c}-\frac{\partial^{2} \Psi_{0}}{\partial a^{2}}=0
$$

where $\mathscr{W}=\omega / \sqrt{g k}$. Therefore, to obtain the $n$ th-order Lagrangian asymptotic solution we first solve the boundary value problems for functions $\Psi_{n}$ in a fixed $(a, c)$-domain with appropriate asymptotic free-surface boundary conditions. Then we construct the $n$-term expansion for $\Psi$, and use the corresponding functions $\xi$ and $\zeta$ to find $(x, z)$ as the limit of the recursion (3.8).

\section{Application to a regular travelling wave in deep water}

The straightforward application of a Stokes-type expansion to the Lagrangian formulation (2.1), (2.3), (2.4), as proposed by Pierson (1962), leads to a non-uniformly valid solution for a regular travelling wave. The corresponding third-order expansions are

$$
\left.\begin{array}{l}
x=a-k \varepsilon \mathrm{e}^{c} \sin (a-t)+(k \varepsilon)^{2} \mathrm{e}^{2 c} t-(k \varepsilon)^{3} \mathrm{e}^{3 c}(2 \sin (a-t)+t \cos (a-t))+\cdots, \\
z=c+k \varepsilon \mathrm{e}^{c} \cos (a-t)+\frac{1}{2}(k \varepsilon)^{2} \mathrm{e}^{2 c}+(k \varepsilon)^{3} \mathrm{e}^{3 c}(\cos (a-t)-t \sin (a-t))+\cdots
\end{array}\right\}
$$

The $(k \varepsilon)^{2}$-term in the expansion for $x$ can be recognized as the Stokes drift and is physically relevant. However, the $(k \varepsilon)^{3}$-terms in the expansions for both $x$ and $z$ include growing secular terms responsible for the solution being non-uniformly valid as $t \rightarrow \infty$. This behaviour can be explained by the nonlinearity of the Lagrangian continuity equation. Due to nonlinear interaction of the second-order non-periodic term corresponding to Stokes drift with a leading term of the expansion, a third-order approximation for the continuity equation will include an unbalanced time-growing right-hand side, and an additional source term with similar behaviour is required in the expansion to compensate for it. The reduction of the continuity equation to the linear form by applying the recursive representation (3.8) should cure this problem. Indeed, it turns out that the $m$ th level of recursion provides the uniformly valid asymptotic solution up to $O(k \varepsilon)^{m+2}$, and with a converging recursion the uniformly valid solution can be constructed for any order of magnitude.

Henceforth we consider solutions with the converged recursion (3.8), that is solutions exactly satisfying the continuity equation (2.1), and we use the phrase 'the order of a solution' for the number of terms in an expansion for $\Psi$. The construction of high-order solutions involves extensive symbolic calculations and requires considerable computational resources, and we restrict our analysis to fifth order. It is convenient to modify the recursive representation (3.8) and explicitly include the non-periodic term representing the Stokes drift as follows:

$$
\alpha_{0}=a+k \varepsilon f(c) t, \quad \gamma_{0}=c,
$$


where function $f(c)$ describes the variation of the mean velocity of the Stokes drift with depth. Fifth-order expansions for the unknown functions are

$$
\Psi(a, c, t)=\sum_{n=0}^{4}(k \varepsilon)^{n} \Psi_{n}(a, c, t)+O(k \varepsilon)^{5}, \quad f(c)=\sum_{n=0}^{4}(k \varepsilon)^{n} f_{n}(c)+O(k \varepsilon)^{5} .
$$

The coefficients of the expansions can be found by back substitution in (3.8), (2.3), (2.4) and expansion of the results for small $k \varepsilon$. Functions $\Psi_{n}$ are $(a ; t)$-periodic solutions of Laplace and Poisson equations, and in the case of deep water they can be represented as linear combinations of terms $\mathrm{e}^{i c} \sin (j(a+t))$, where $i$ and $j$ are integers. When $i=j$ we have a regular wave solution of the Laplace equation, and terms with $i \neq j$ are used to balance non-homogeneous terms in equations arising at higher orders. The periodic part of the solution is found to be

$$
\left.\begin{array}{l}
\Psi_{0}=\mathrm{e}^{c} \sin (a-t), \quad \Psi_{1}=0, \quad \Psi_{2}=\frac{5}{8} \mathrm{e}^{3 c} \sin (a-t), \\
\Psi_{3}=\left(\frac{1}{4} \mathrm{e}^{2 c}-\frac{5}{24} \mathrm{e}^{4 c}\right) \sin (2(a-t)), \\
\Psi_{4}=\left(\frac{3}{4} \mathrm{e}^{3 c}+\frac{39}{32} \mathrm{e}^{5 c}\right) \sin (a-t)+\left(\frac{1}{36} \mathrm{e}^{3 c}+\frac{49}{1152} \mathrm{e}^{5 c}\right) \sin (3(a-t)) .
\end{array}\right\}
$$

Functions $f_{n}$ balance the non-periodic terms of the equations, and the corresponding expansion is

$$
f(c)=(k \varepsilon) \mathrm{e}^{2 c}+2(k \varepsilon)^{3} \mathrm{e}^{4 c}+O(k \varepsilon)^{6} .
$$

The free-surface condition (2.4) can be satisfied only for a specific value of the dispersion parameter $\mathscr{W}$, leading to the following expansion for the dispersion relation:

$$
\mathscr{W}=\frac{\omega}{\sqrt{g k}}=1+\frac{1}{2}(k \varepsilon)^{2}+\frac{9}{8}(k \varepsilon)^{4}+O(k \varepsilon)^{6} .
$$

Functions $\Psi_{n}$ are bounded at any time and for any value of their first argument. The infinitely increasing term in (5.2) leads to the unbounded growth of $\alpha_{m}$ in (3.8) while $\gamma_{m}$ remains bounded. Together with the convergence of the recursion (3.8) this means that the solution for the vertical coordinate $z$ remains bounded and the entire solution is uniformly valid at all times.

The parameter $k \varepsilon$ has no clear physical meaning outside our method of solution. To compare our results with results obtained by other methods it is convenient to use wave steepness $k A \rightarrow 0$ or $A / \lambda \rightarrow 0$ as a new small parameter, where $\lambda=2 \pi / k$ is wavelength and

$$
A=\varepsilon(z(0,0,0)-z(\pi, 0,0)) / 2
$$

is wave amplitude. These parameters are more physically relevant and independent of the particular form of the solution. The asymptotic expansion of $A$ with respect to $k \varepsilon$ is

$$
A=\varepsilon\left(1+\frac{1}{2}(k \varepsilon)^{2}+\frac{9}{8}(k \varepsilon)^{4}+O(k \varepsilon)^{6}\right),
$$

and all results can now be re-expanded by using these new parameters. For example, the expansion for the square of the phase velocity is

$$
C^{2}=(\omega / k)^{2}=\frac{g}{k}\left(1+(k A)^{2}+\frac{1}{2}(k A)^{4}+O(k A)^{6}\right),
$$

which reproduces the result obtained from the Stokes expansion (e.g. Schwartz 1974).

Expansions (5.3-5.5) can be interpreted in a dual way: (i) as low-order asymptotic expansions for $(k \varepsilon \rightarrow 0)$; (ii) as the first few terms of an infinite series, which in the case of convergence for $n \rightarrow \infty$ represents an exact solution. In the latter case 
there is no assumption on the smallness of the expansion parameter. A classical example of such a duality is the Stokes expansion. Originally a low-order expansion was obtained as an asymptotic solution for small wave steepness. However, after the proof of convergence of the expansion for the whole range of wave amplitudes up to the limiting one given by Levi-Cività (1925) and Krasovskii (1960), it became possible to consider expansions truncated at large $n$ as exact solutions calculated with a certain accuracy (e.g. Schwartz 1974; Cokelet 1977). Comparison of the few first approximations with the exact solution can give an insight into a possible solution behaviour for large $n$. Figures $2-5$ show selected examples of such a comparison.

Applying the arguments used for the model solution (3.9) we can obtain critical values of the steepness parameter $k \varepsilon$ and crest elevations of limiting profiles of asymptotic solutions of different orders. Without loss of generality we perform this analysis at $t=0$. The first-order solution then coincides with the model solution (3.9), and we obtain all results in the closed analytical form. For more complicated third- and fifth-order solutions, transcendent algebraic equations analogous to (3.10) are solved by iteration. For the first-, third- and fifth-order asymptotic solutions we found the critical values of $k \varepsilon$ to be $0.736,0.459$ and 0.400 respectively, and the corresponding values of $z$ at wave crests are $0.318,0.196,0.164$. The direct calculations of the recursive solutions give identical results. Figure 2(a) shows limiting profiles corresponding to different orders of the asymptotic solution compared with the exact limiting profile from Williams (1981). In $\S 2$ we have shown that the critical profile of the model solution has a cusp at the wave crest. The same applies to the firstorder asymptotic solution. We do not prove this result for the third- and fifth-order solutions, but referring to figure 2, we can suggest that they also have a similar singularity at the crests of limiting waves, which differs from the corner at the crest of the actual limiting wave. Apart from this, the shape of the asymptotic limiting profiles is reasonable and approaches the exact limiting profile as the order of the asymptotic solution increases. The asymptotic wave profiles of different orders with the steepness equal to the steepness of the actual limiting wave $A / \lambda=0.0706$ also converge to the exact solution, as can be seen on figure $2(b)$. Horizontal velocity profiles for different orders of asymptotic solution for a wave of steepness $A / \lambda=0.0706$ shown on figure 3 also demonstrate convergence to the exact solution for the limiting wave calculated by Williams (1981). The agreement between the exact and asymptotic solutions of high orders is quite good apart from the region of the highest volume deformation near the wave crest. Figure 4 shows the dependence of the densities of the kinetic and potential energies on the wave steepness for asymptotic solutions, in comparison with the exact result of Longuet-Higgins (1975). There is an excellent agreement for waves of small and moderate steepness and improvement of the high-amplitude results for higher orders of asymptotic solution. The fifth-order solution provides a very good agreement for both $T$ and $V$ for amplitudes up to $A / \lambda=0.06$. The exact solution has an energy maximum below the highest wave, whereas the asymptotic solutions do not have this property and reach maximum energy at their, significantly higher, limiting solutions.

Although we consider an irrotational flow, we have zeros only for the first few terms of an asymptotic expansion for the vorticity distribution $\Omega(a, c)$, and non-zero terms appear at higher orders. The distribution of this non-physical vorticity for different orders of the Lagrangian asymptotic solution is presented in figure 5. For the wave of moderate amplitude $(A / \lambda=0.05)$ a considerable reduction of unphysical vorticity for higher-order solutions can be clearly observed. For a high wave amplitude $(A / \lambda=0.0706)$ in the region of strong local fluid deformation near the free surface 


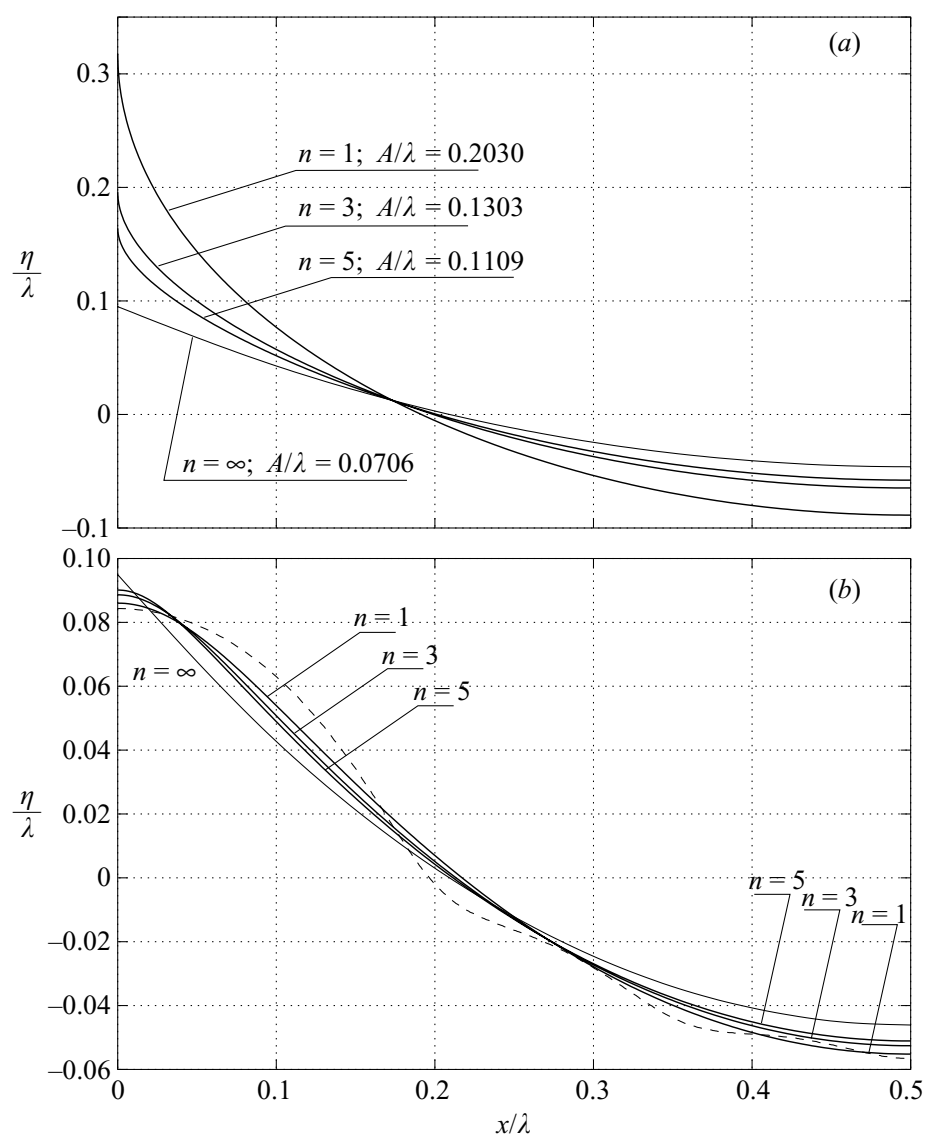

FIGURE 2. (a) Convergence of the limiting profiles for different orders of the Lagrangian asymptotic solution to the actual limiting profile, and $(b)$ convergence of the wave profiles with steepness $A / \lambda=0.0706$ for different orders of the Lagrangian asymptotic solution to the actual limiting profile. The dashed line represents the profile for a fifth-order Stokes expansion with $A / \lambda=0.0706 . n=\infty$ refers to the actual limiting profile as presented by Williams (1981).

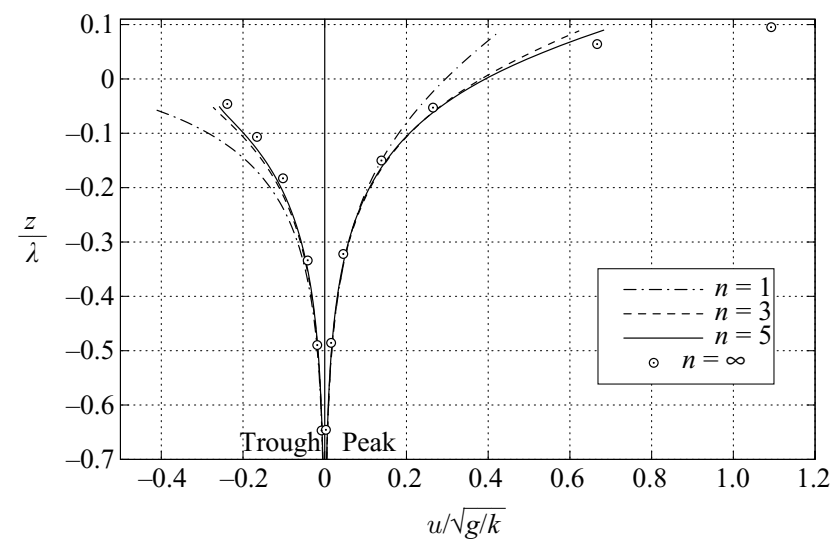

FIGURE 3. Profiles of horizontal velocity under troughs and peaks of a regular travelling wave of the limiting steepness $A / \lambda=0.0706$ for different orders of the Lagrangian asymptotic solution. $n=\infty$ refers to the exact solution for the limiting wave as presented by Williams (1981). 


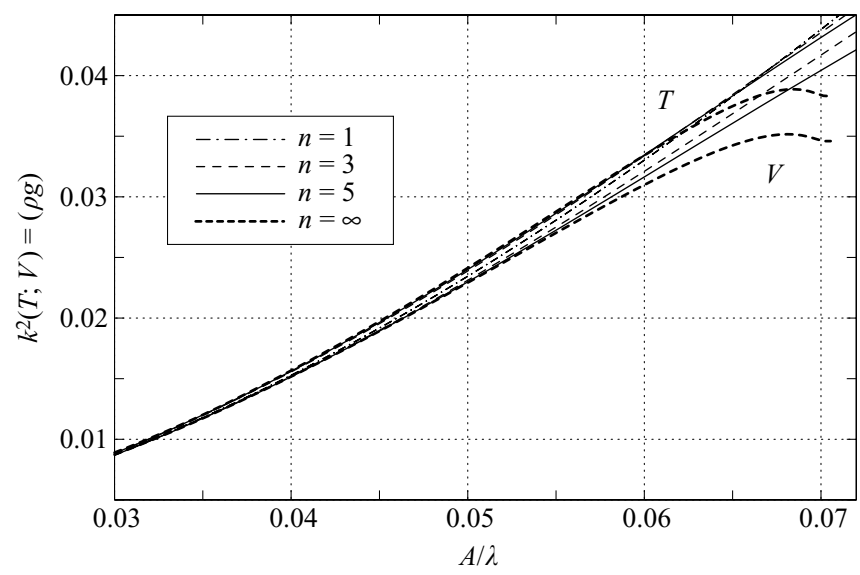

FiguRE 4. The densities of the kinetic $(T)$ and potential $(V)$ energies as functions of the wave amplitude for different orders of the Lagrangian asymptotic solution. $n=\infty$ refers to an exact solution as presented by Longuet-Higgins (1975).

the improvement is not so obvious. However, this occurs in a region near the free surface, which becomes narrower for higher-order solutions due to faster exponential decay of the vorticity with depth.

\section{Discussion}

The principal result of the paper is the reduction of the Lagrangian continuity equation (2.1) to the linear Eulerian form (3.4). This result is quite general and can be applied to any two-dimensional continuous flow. We have demonstrated that the mean point $(\alpha, \gamma)$ between the original and final points of an area-preserving mapping $(a, c) \rightarrow(x, z)$ satisfying $(2.1)$ can be represented as a fixed point of a two-dimensional transformation (3.7). Components $(\xi, \zeta)$ of a transformation function satisfy the linear continuity equation (3.4) and can be expressed via a single differentiable function $\Psi$ (3.5). Not all functions $\Psi$ result in a fixed point and therefore an area-preserving mapping. Various fixed-point theorems (e.g Zeidler 1998) can be applied to specify sufficient conditions of existence of a fixed point for any particular $\Psi$. We give an example of the application of a one-dimensional fixed-point theorem to a simple model solution (3.9). The condition (3.6) of the regularity of a mapping $(a, c) \rightarrow(\alpha, \gamma)$ and the convergence of the recursion (3.8) also provide sufficient conditions for the existence of an area-preserving mapping. We should emphasize that all conditions discussed here are sufficient and breaking of any of them does not mean that the mapping does not exist. An important question is the physical meaning of functions $\Psi$ which do not generate an area-preserving mapping for the whole domain occupied by fluid. A possible example is the model solution (3.9) with an amplitude higher than the critical one. It is clear that flows with smooth solid boundaries always remain continuous and a properly formulated dynamical problem for $\Psi$ does not have such solutions. For flows with a free surface, however, phenomena involving the loss of surface continuity are possible, such as jet formation on a crest of a high-amplitude standing wave (Bredmose et al. 2003), or spilling breaking of a steep travelling wave in deep water (Banner \& Peregrine 1993). Therefore, for free-surface flows the dynamical evolution of $\Psi$ can potentially lead to situations when in certain regions of a fluid 


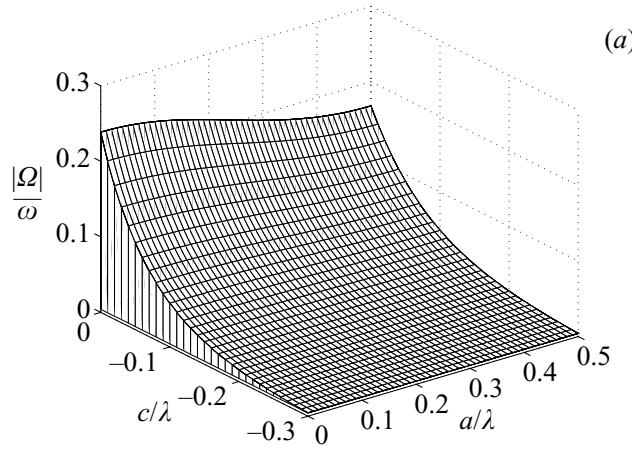

(a)

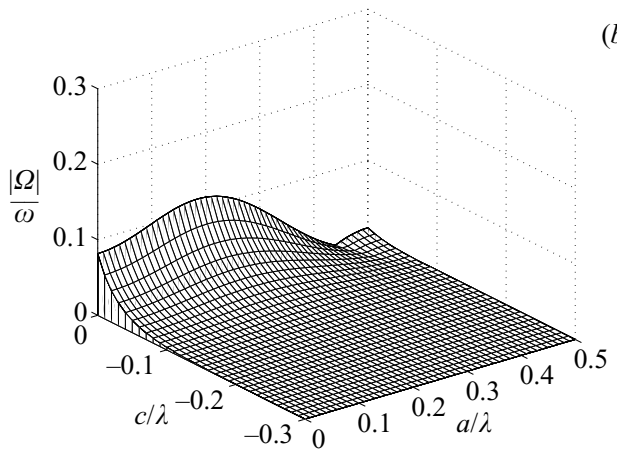

(b)
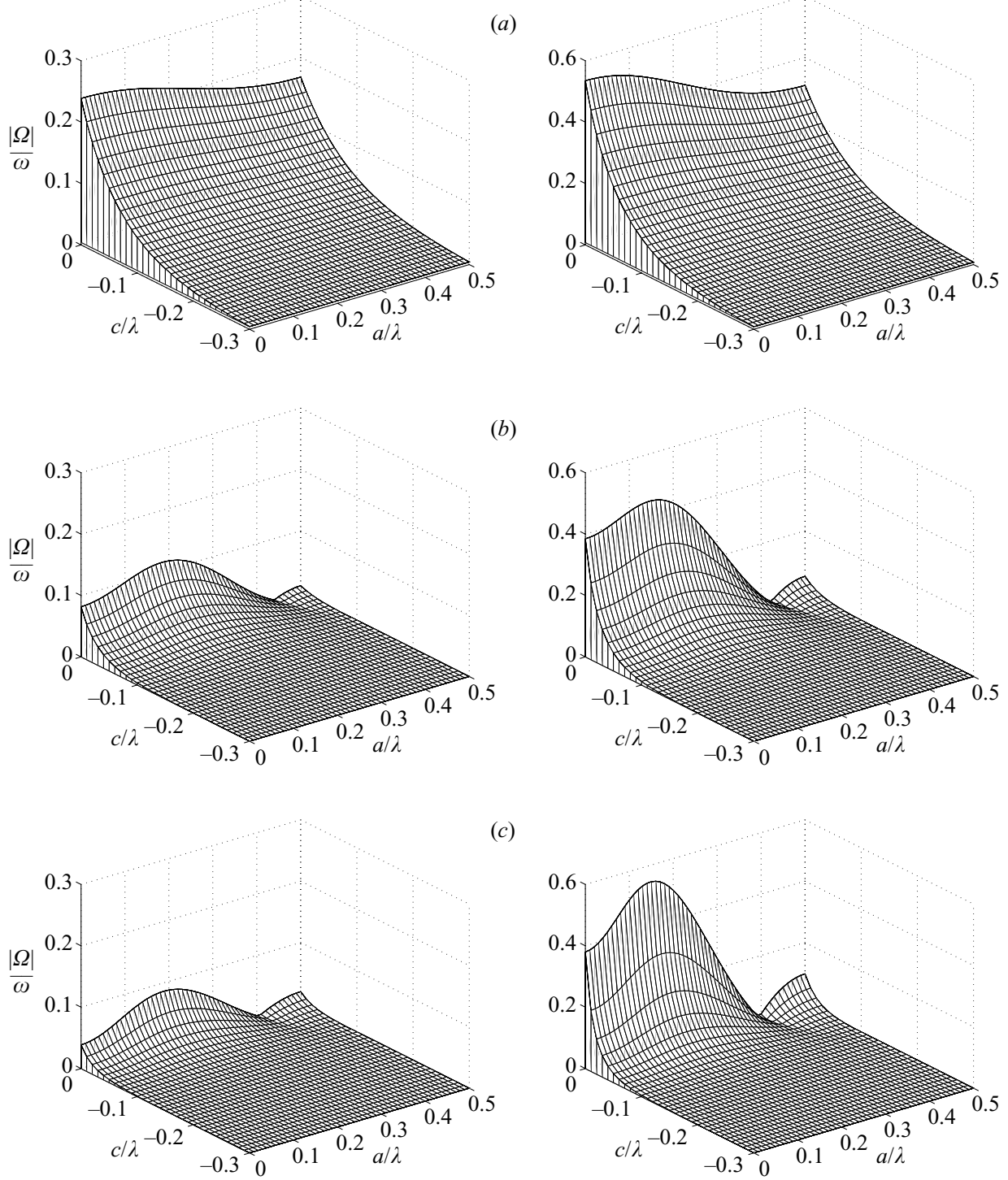

(c)

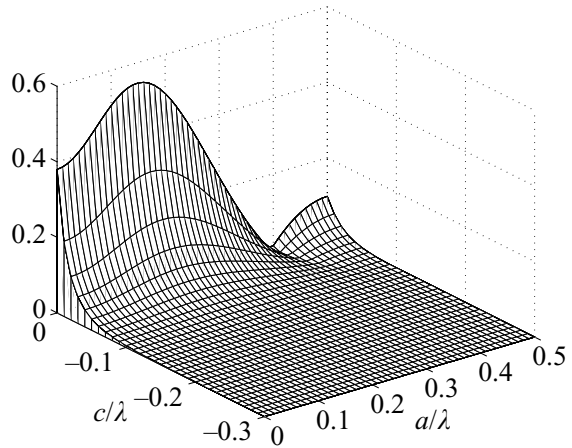

FIGURE 5. The absolute value of vorticity generated by the neglected terms of the asymptotic expansion for $(a)$ the first, $(b)$ third and $(c)$ fifth orders of the Lagrangian asymptotic solution. $A / \lambda=0.05$ (left) and $A / \lambda=0.0706$ (right).

domain it will not generate an area-preserving mapping, and we expect that such solutions can be associated with physical flow discontinuities.

Regarding a regular travelling wave, we have obtained the first few approximations of a uniformly valid small-amplitude asymptotic solution. These approximate solutions can at the same time be the leading terms of an infinite sequence, which for $n \rightarrow \infty$ converges to an exact solution for a finite wave amplitude. We cannot prove the convergence of the whole sequence as $n \rightarrow \infty$. However, by analysing the first few terms we can suggest the whole sequence behaviour for large $n$. Fast convergence takes place for regions of small local deformation of fluid volume. This can be clearly observed for internal points on the plots of figure 5, especially for 
a small-amplitude solution. The situation is not so obvious for strongly deformed regions near the free surface. For example, asymptotic limiting profiles have cusps at wave crests (figure $2 a$ ). This significantly differs from a corner at the crests of an actual limiting wave. Nevertheless, for higher-order solutions the region where the local steepness of an asymptotic profile grows rapidly and generates the cusp shrinks to a small vicinity around the wave crest. The shape of profiles outside this small region is physically reasonable and approaches the actual limiting profile as the order of the asymptotic solution increases. The structure of the vorticity of large-amplitude asymptotic solutions (e.g. figure 5, right-hand column) suggests possible non-uniform convergence, when the value of vorticity in a region near the free surface does not vanish as $n \rightarrow \infty$. The region, however, becomes thinner as $n$ grows, and in the limit it could form a vortex sheet on the free surface. Altogether, the results show that the entire sequence of approximate solutions can converge for increasing $n$. We can suggest that uniform convergence takes place for $x$ and $z$, while the convergence of their spatial derivatives on the free surface is not uniform for large wave amplitudes. Regardless of the convergence of expansions for $n \rightarrow \infty$, our solutions are consistent if treated as low-order asymptotic approximations for small $k \varepsilon$.

There is a close link between the recursive solution (3.8), (5.3)-(5.5) and a Stokestype Lagrangian expansion (5.1). Both methods provide the same expansions for the mean velocity of the Stokes drift and for the dispersion relation. The $n$ th-order Stokes-type expansion can be obtained as an expansion of the $n$ th- or higher-order recursive solution in a series for small $k \varepsilon$. This is not surprising, for these are two asymptotic representations of the same solution, and they differ by the type of convergence for large $n$. The convergence of a Stokes-type Lagrangian expansion as $n \rightarrow \infty$ (if it exists) is not uniform at large times. Any finite sum $(n>3)$ of the expansion for surface elevation increases without limit as $t \rightarrow \infty$. This is why for $k \varepsilon \rightarrow 0$ any expansion with a finite number of terms is non-uniformly valid for large $t$ in a conventional asymptotic sense. On the other hand, an asymptotic expansion for $\Psi$ (5.3) is a bounded function of $t$ and $a$. Together with the convergence of recursion (3.8) this provides the boundedness of vertical displacement for any $n$, which means that for small $k \varepsilon$ the solution for surface elevation is uniformly valid for large $t$.

An important question is the possibility of generalizing our results to three dimensions. A general three-dimensional vector-function satisfying the linear continuity equation cannot be expressed in terms of a scalar stream function, and three components of a vector potential are required instead. A direct utilization of a three-dimensional analogue of the recursive form (3.8) for representing components of a vector potential leads to a solution which satisfies the continuity equation only to $O(k \varepsilon)^{2}$. An analysis similar to that of $\S 2$ shows that three-dimensional transformed coordinates analogous to (3.2) cannot depend only on particle coordinates, but will include derivatives with respect to the Lagrangian labels. This makes the construction of a three-dimensional recursive solution rather problematic, even if a three-dimensional analogue of (3.2) exists, because fixed-point iterations will not be applicable. Therefore, a generalization of the results to three dimensions is not straightforward and requires further investigation.

\section{REFERENCES}

Banner, M. L. \& Peregrine, D. H. 1993 Wave breaking in deep water. Annu. Rev. Fluid Mech. 25, 373-397.

Bredmose, H., Brocchini, M., Peregrine, D. H. \& Thais, L. 2003 Experimental investigation and numerical modelling of steep forced water waves. J. Fluid Mech. 490, 217-249. 
Cokelet, E. D. 1977 Steep gravity waves in water of arbitrary uniform depth. Phil. Trans. R. Soc. Lond. A 286, 183-230.

GJøsund, S. H. 2003 A Lagrangian model for irregular waves and wave kinematics. Trans. ASME: J. Offshore Mech. Arctic Engng 125, 94-102.

KrasovskiI, Y. P. 1960 On the theory of steady waves of not small amplitude. Dokl. Akad. Nauk. SSSR 130, 1237 (in Russian).

Lamb, H. 1932 Hydrodynamics. Cambridge University Press.

LEVI-CivitÀ, T. 1925 Determination rigoureuse des ondes permanentes d'ampleur finie. Math. Ann. 93, 264-314.

Longuet-Higgins, M. S. 1975 Integral properties of periodic gravity waves of finite amplitude. Proc. R. Soc. Lond. A 342, 157-174.

Longuet-Higgins, M. S. 1979 The trajectories of particles in steep, symmetric gravity waves. J. Fluid Mech. 94, 497-517.

Longuet-Higgins, M. S. 1986 Eulerian and Lagrangian aspects of surface waves. J. Fluid Mech. 173, 683-707.

LonguET-HigGins, M. S. 1987 Lagrangian moments and mass transport in Stokes waves. J. Fluid Mech. 179, 545-555.

MCINTYRE, M. E. 1988 A note on the divergence effect and the Lagrangian-mean surface elevation in water waves. J. Fluid Mech. 189, 235-242.

Pierson, W. J. 1962 Perturbation analysis of the Navier-Stokes equations in Lagrangian form with selected linear solutions. J. Geophys. Res. 67, 3151-3160.

Salmon, R. 1988 Hamiltonian fluid mechanics. Annu. Rev. Fluid Mech. 20, 225-256.

SChwartz, L. W. 1974 Computer extension and analytic continuation of Stokes' expansion for gravity waves. J. Fluid Mech. 62, 553-578.

Schwartz, L. W. \& Fenton, J. D. 1982 Strongly nonlinear waves. Annu. Rev. Fluid Mech. 14, 39-60.

Stokes, G. G. 1847 On the theory of oscillatory waves. Trans. Camb. Phil. Soc. 8, 441-455.

ÜnLÜATA, Ü. \& MeI, C. C. 1970 Mass transport in water waves. J. Geophys. Res. 75, 7611-7618.

Williams, J. M. 1981 Limiting gravity waves in water of finite depth. Phil. Trans. R. Soc. Lond. A 302, 139-188.

ZeIdler, E. 1998 Nonlinear Functional Analysis and its Applications, Vol. 1, Fixed-Point Theorems. Springer. 